\title{
Osificación del cartílago costal de la primera costilla en relación con la edad. Aplicaciones en la estimación forense de la edad.
}

\author{
Ossification of first rib cartilage related to age. Applications \\ in the forensic estimation of age.
}

\author{
PM. Garamendi' ${ }^{1}$, MI. Landa², I. Alemán ${ }^{3}$ y MC. Botella ${ }^{3}$
}

\begin{abstract}
RESUMEN
Se presenta un estudio sobre una muestra de 123 radiografías digitales de tórax de sujetos entre 5 y 75 años de edad en las que se ha analizado el estado de osificación del cartílago costal de la primera costilla. En este estudio el estadio 0 de osificación suele asociarse con edades entre 5 y 19 años. Los estadios de osificación 2 y 3 se han asociado con edades mínimas de 24 años de edad. Autores previos (Michelson, 1934) ya habían indicado que la edad mínima a la que los sujetos estudiados presentaban un estadio 3 de osificación era de 2 laños de edad. Se sugiere la posibilidad de integrar el estudio de la osificación de la primera costilla entre los datos diagnósticos manejados para la estimación forense de edad en sujetos vivos basada en las recomendaciones del AGFAD del año 2000.
\end{abstract}

Palabras clave: Primera costilla; osificación; cartílago costal; edad; estimación forense de la edad.

Cuad Med Forense 2007; 13(50):243-253

\section{ABSTRACT}

The results of a research on ossification of first rib cartilage in a Spanish population are presented in this paper. Our sample is constituted by 123 digital $x$-rays studies had made up our sample. Minimum subject age has been 5 years old and maximum age 75 years old. Stage 0 has been usually found out in subjects between 5 and 19 years of age. Stages 2 and 3 had been found out in subjects at a minimum age of 24 years old. Previous researches had pointed out that stage 3 could be observed in subjects at an age of at least 21 years of age. We suggest that the analysis of the ossification degree of the first rib cartilage could be useful as a complementary method for the forensic estimation of age based on radiographic methods.

Key words: First rib; ossification; costal cartilage; age; forensic age estimation.

Trabajo parcialmente financiado por el Ministerio de Educación y Ciencia (ref. TIN2006-00829) y por la Consejería de Innovación, Ciencia y Empresa de la Junta de Andalucía (ref. TIC1619), ambos con fondos FEDER.

Correspondencia: Pedro Manuel Garamendi. Instituto de Medicina Legal de Huelva. Plaza Isabel La Católica, 9. 21071. Huelva. E-mail: imanolgaramendi@gmail.com.

${ }^{1}$ Médico Forense. IML de Huelva.

2 Médico Forense. IVML. Subdirección de Vizcaya.

3 Antropólogo Forense. Laboratorio de Antropología Física. Universidad de Granada. 


\section{INTRODUCCIÓN:}

En septiembre de 2.000, el grupo multidisciplinario del Arbeitsgemeinschaft für Forensische Altersdiagnostik der Deutschen Gesellschaft für Rechtsmedizin (AGFAD) elaboró una de las primeras guías para la estimación de la edad en individuos vivos sometidos a procedimientos criminales $[1,2,3]$. Las guías establecían la necesidad de realizar una serie de pruebas diagnósticas que incluyeran exámenes radiográficos y antropométricos y, una vez practicadas éstas, interpretar los resultados en función de estudios previos en poblaciones adecuadas al sujeto de estudio.

Estas guías recomiendan, para la determinación de la mayoría o minoría de edad penal ( 8 años) en sujetos vivos, la práctica de las siguientes pruebas:

I. Examen físico: obtención de medidas antropométricas (peso, talla, tipo constitucional); inspección de signos de madurez sexual; identificación de patologías que puedan alterar el desarrollo madurativo.

2. Examen radiográfico de la mano izquierda.

3. Examen externo del estado de la dentición y radiografía dental.

4. Examen radiográfico de la clavícula, en el caso de que interesase confirmar si la edad cronológica es mayor o menor de 21 años de edad.

Al interpretar los resultados, las mismas guías recomiendan que los datos obtenidos de las anteriores pruebas sean contrastados con estudios de referencia adecuados al sujeto específico estudiado. Al seleccionar dichos estudios debieran considerarse como factores significativos de corrección de los resultados las variables étnicas, socioeconómicas y patológicas, así como el grado de error intra e interobservador. Recomiendan, finalmente, al realizar la valoración pericial definitiva que se especifique el resultado por separado de cada una de las pruebas realizadas y que la estimación de edad se establezca como la más probable, indicando el grado de probabilidad del resultado estimado.

La osificación de la región condral de la primera costilla ha sido objeto de algunos estudios previos que ponen de manifiesto su carácter progresivo con la edad. El presente estudio pretende presentar las conclusiones de una investigación realizada sobre radiografías digitales de tórax en las que se ha analizado el proceso de osificación del cartílago costal de la primera costilla con la edad. Este parámetro de maduración con la edad puede ser cuantificado en radiografías de tórax posteroanteriores, las mismas en las que se puede evaluar también la región de la extremidad esternal de la clavícula, parámetro recomendado por el AGFAD para el diagnóstico de edad entre los 18 y 21 años de edad. Sería posible, por tanto, añadir la valoración del grado de osificación de este cartílago entre los parámetros de diagnóstico de edad recomendables para establecer este diagnóstico en sujetos en torno a 20 años de edad sin necesidad de ampliar las pruebas radiográicas diagnósticas realizadas según las recomendaciones del AGFAD en sujetos vivos.

\section{MATERIAL Y MÉTODOS:}

Para este estudio se han analizado 123 radiografías de tórax postero-anteriores originalmente obtenidas en formato digital. La distribución demográfica por edades y sexos de estas radiografías se incluye en las tablas 1 y 2.

Las imágenes radiográficas digitalizadas han sido facilitadas amablemente por un centro de radiodiagnóstico privado de Bilbao (Preteimagen) y la muestra de población de las que se han seleccionado las imágenes corresponde con una muestra aleatoria de sujetos estudiados en dicho centro. Las radiografías han sido realizadas tanto a sujetos que presentan alteraciones patológicas en 
la región anatómica seleccionada, como a sujetos sometidos a estas pruebas con carácter rutinario en pruebas de screening de población general o en el curso de estudios preoperatorios por otras patologías. Del total original de 341 radiografías de tórax se seleccionaron 123 según criterios de definición homogénea de grupos interanuales de edad y criterios de calidad relativa de las imágenes radiográficas.

Las imágenes digitales de las radiografías se

\begin{tabular}{|c|ccc|}
\hline Edad & Hombres & Mujeres & Total \\
\hline $0-20$ & 12 & 12 & 24 \\
$20-30$ & 20 & 20 & 40 \\
$30-40$ & 20 & 20 & 40 \\
$40-50$ & 20 & 20 & 40 \\
$50-60$ & 20 & 20 & 40 \\
$60-70$ & 20 & 20 & 40 \\
$>70$ & 10 & 12 & 22 \\
Total & 122 & 124 & 246 \\
\hline
\end{tabular}

Tabla 1. Radiografías de tórax por grupos de edad y sexo analizadas. obtuvieron originalmente en formato DICOM. No obstante, dado el tamaño relativo de estas imágenes y la limitación de funciones de uso que se iba a hacer de las mismas, se decidió transformarlas a formato JPG. De este modo, las características de identificación de la imagen radiográfica y otras funciones incluidas en el formato DICOM se eliminaron, pero el

\begin{tabular}{|c|c|c|c|c|c|c|}
\hline $\begin{array}{c}\text { Grupos } \\
\text { Edad }\end{array}$ & Media & $\mathrm{N}$ & $\begin{array}{c}\text { Desviación } \\
\text { standard }\end{array}$ & Minimo & Máximo & Mediana \\
\hline $0-20$ & 15,972603 & 24 & 4,4029583 & 5,3479 & 19,7205 & 17,732877 \\
$20-30$ & 25,159932 & 40 & 2,8114657 & 20,0904 & 29,5973 & 25,179452 \\
$30-40$ & 35,264110 & 40 & 2,8953552 & 30,6658 & 39,9644 & 35,078082 \\
$40-50$ & 45,031644 & 40 & 2,7420453 & 40,4027 & 49,2411 & 45,517808 \\
$50-60$ & 55,192466 & 40 & 2,6385442 & 51,4384 & 59,9041 & 54,980822 \\
$60-70$ & 64,293288 & 40 & 2,6016542 & 60,2082 & 69,3151 & 64,382192 \\
$70-80$ & 72,694645 & 22 & 1,8292717 & 70,2740 & 75,4110 & 72,816438 \\
Total & 44,635293 & 246 & 17,7998784 & 5,3479 & 75,4110 & 45,424658 \\
\hline
\end{tabular}

tamaño relativo de los archivos se redujo. Antes de proceder a su modificación, se realizó un test con varias radiografías digitales en formatos DICOM transforma-

Tabla 2. Medidas estadísticas de distribución de edades en la muestra de radiografías de tórax de ambos sexos. Las cifras de número de casos expresan los valores de radiografías de cada hemicuerpo analizadas.

das a JPG y se pudo comprobar que para

los fines del análisis que se iba a realizar sobre las mismas, la calidad de la imagen no se alteraba. La resolución final de las radiografías de tórax fue de 2140 × 1760 píxeles a 8 bits de escala de grises.

Estas imágenes digitales en formato JPG se han analizado mediante la aplicación de las funciones de un programa de tratamiento de imágenes específico: Image-J I.34s (versión más actual v. I.38) [4]. Este es un programa de tratamiento de imagen gratuito, de código abierto y multiplataforma que permite la visualización de imágenes radiográficas y su manipulación. Se hizo un análisis previo del uso de este programa informático frente a otras herramientas informáticas de tratamiento de imagen digital aplicadas por autores previos, como el programa Adobe Photoshop CS y CS2, que precisa uso con licencia, o el programa GIMP, también gratuito y de código abierto. Pudo comprobarse que para los fines buscados en este estudio, de simple inspección de contornos óseos utilizando las funciones de modificación de características de brillo y contraste o restricción de escala de grises disponibles en estos programas, no se encontraron diferencias significativas entre el uso de uno u otro tipo de programa.

En estas radiografías de tórax se ha analizado el aspecto morfológico de la región del cartílago costal de la primera costilla en ambas primeras costillas según un sistema de estadios equivalente a los estadios de osificación propuestos por Barchilon y Michelson $[5,6]$. Se incluyen ejemplos de cada uno de los estadios descritos por Barchilon y Michelson en las imágenes I a 4. 


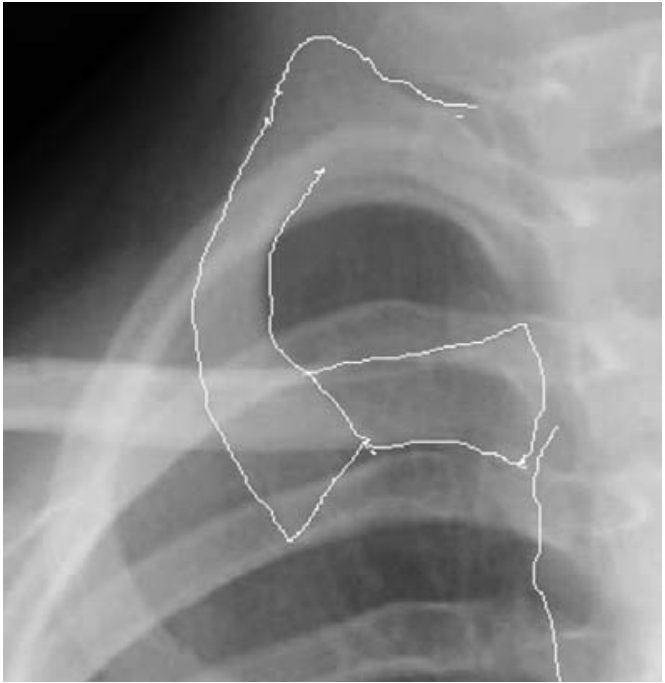

Imagen 1. Osificación del cartílago costal de la primera costilla. Estadio 0 de Barchilon, equivalente a no osificación.

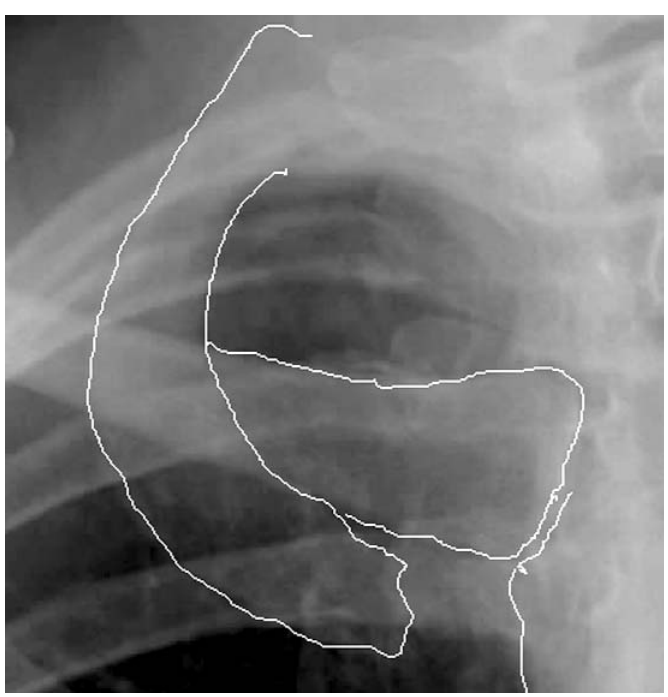

Imagen 3. Osificación del cartílago costal de la primera costilla. Estadio 2 de Barchilon, equivalente a osificación en torno al $50 \%$ del cartílago.

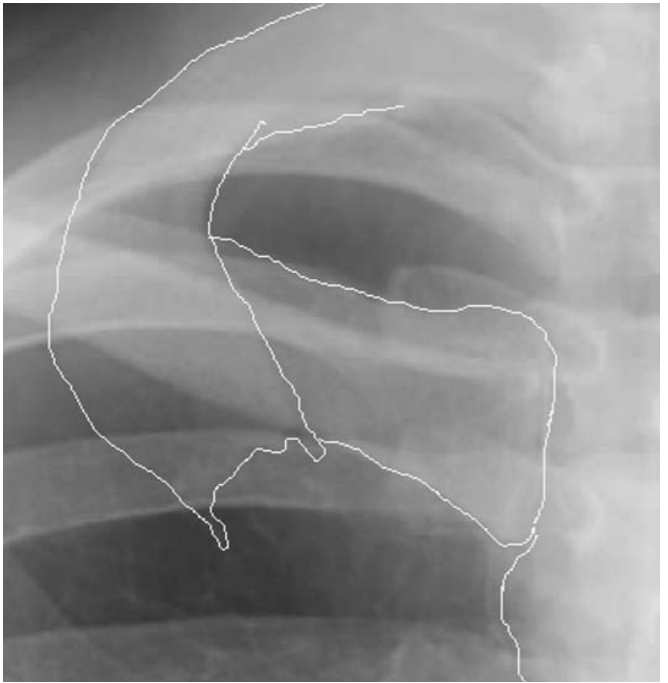

Imagen 2. Osificación del cartílago costal de la primera costilla. Estadio 1 de Barchilon, equivalente a osificación incipiente.

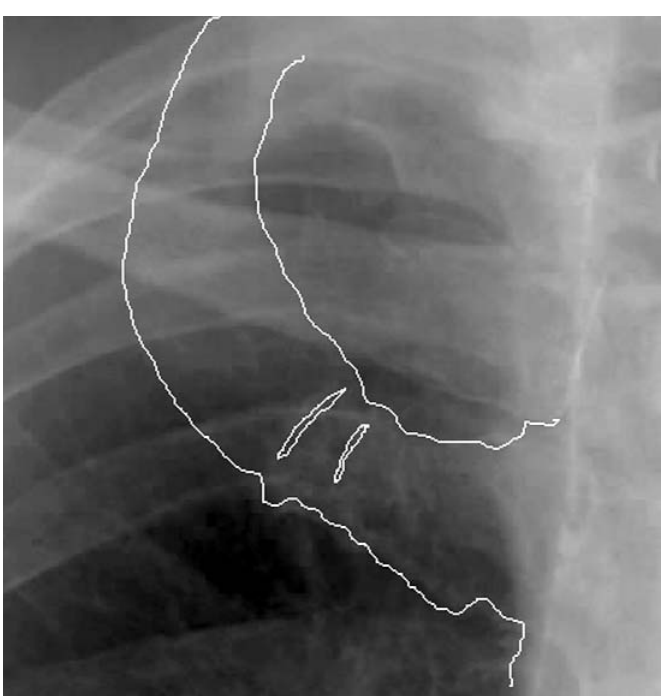

Imagen 4. Osificación del cartílago costal de la primera costilla. Estadio 3 de Barchilon, equivalente a osificación completa o casi completa.

Al tratarse de una serie en la que se comparan valores cualitativos (valores de estadios de osificación del cartílago costal descritos por Barchilon) y cuantitativos (edad), los estadísticos analizados han incluido medidas estadísticas de distribución generales: media aritmética, desviación estándar, mediana, valores máximos y valores mínimos. Las medidas se han analizado por grupos de edad y por sexos. Gráficamente los resultados se han expresado mediante gráficos de cajas y bigotes (box-plot) (Gráficos I a 3). 


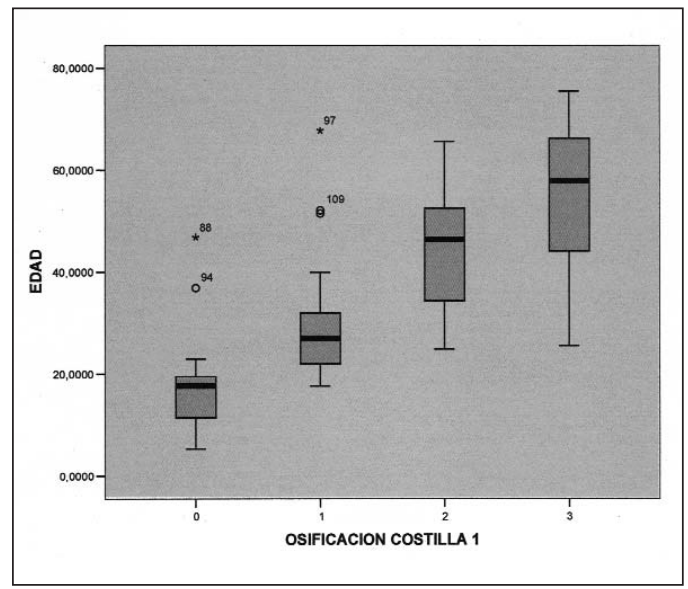

Gráfico 1. Diagrama de cajas y bigotes para osificación de primera costilla en ambos sexos (Estadios de Barchilon).

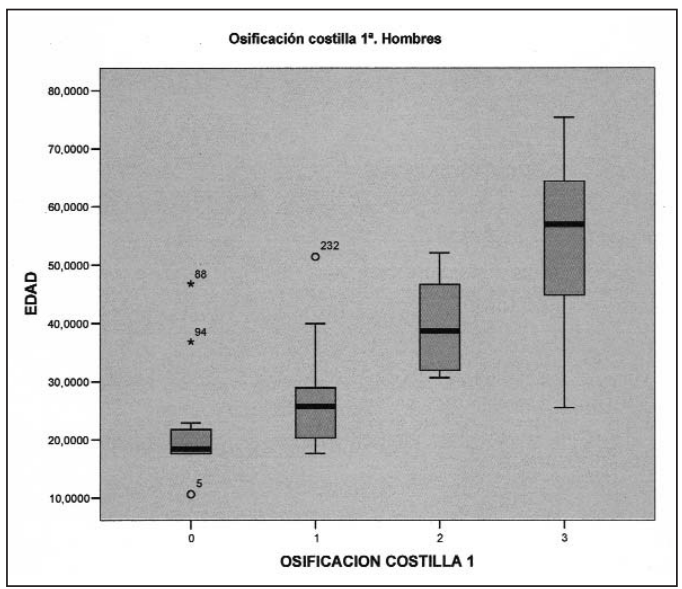

Gráfico 2. Diagrama de cajas y bigotes para osificación de primera costilla en hombres (Estadios de Barchilon).

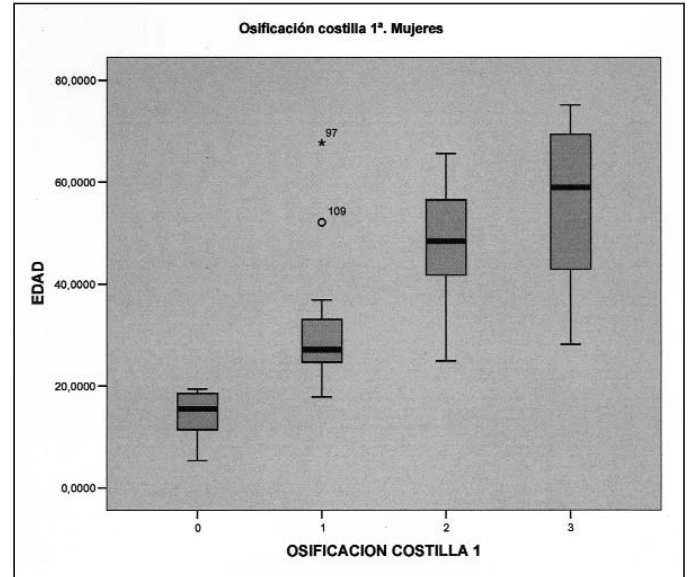

Gráfico 3. Diagrama de cajas y bigotes para osificación de primera costilla en mujeres (Estadios de Barchilon).

Para intentar aproximar un cálculo de correlación entre los resultados y la edad se ha considerado también la variable de estadio de Barchilon como una variable continua y se ha obtenido el coeficiente de correlación de Pearson para ambos sexos.

Para valorar la posible independencia entre variables cualitativas se ha utilizado la Prueba del Chi Cuadrado de Pearson sobre tablas de contingencia.

\section{RESULTADOS:}

La muestra de población de sujetos de los que se obtuvieron las radiografías de tórax se distribuyeron según se indica en las tablas 1 y 2 . La media de edad de los sujetos de ambos sexos fue de 44,63 con unas edades máximas y mínimas de 5,3 y 75,4 años de edad.

Los resultados generales de distribución de edades y sexos según la identificación en las radiografías de cada uno de los estadios de Michelson y Barchilon se incluyen en las tablas 3, 4 y 5. En las tablas 6 y 7 se recogen los resultados de distribución de los citados estadios según lateralidad. 
El estadio 0 se ha observado en sujetos entre 5,3 y 46,8 años de edad, en mujeres hasta los 19,4 años de edad. Los estadios 2 y 3 se han observado en sujetos de edades mínimas de 24,8 años de edad para mujeres y 25,4 años en hombres.

\begin{tabular}{|c|c|c|c|c|c|c|}
\hline $\begin{array}{c}\text { OSIFICACION } \\
\text { COSTILLA 1 }\end{array}$ & Media & N & $\begin{array}{c}\text { Desviación } \\
\text { Standard }\end{array}$ & Minimo & Máximo & Mediana \\
\hline 0 & 18,032877 & 21 & 9,3582139 & 5,3479 & 46,8274 & 17,641096 \\
1 & 28,087513 & 52 & 9,2336348 & 17,6082 & 67,7233 & 26,882192 \\
2 & 45,742180 & 48 & 11,5735603 & 24,8740 & 65,5918 & 46,454795 \\
3 & 55,563332 & 125 & 13,0576578 & 25,4849 & 75,4110 & 57,276712 \\
Total & 44,635293 & 246 & 17,7998784 & 5,3479 & 75,4110 & 45,424658 \\
\hline
\end{tabular}

Tabla 3. Distribución de resultados de edades en relación con el sistema de estadios de Barchilon para la primera costilla en ambos sexos.

\begin{tabular}{|c|c|c|c|c|c|c|}
\hline $\begin{array}{c}\text { OSIFICACION } \\
\text { COSTILLA 1 }\end{array}$ & Media & N & $\begin{array}{c}\text { Desviación } \\
\text { Standard }\end{array}$ & Minimo & Máximo & Mediana \\
\hline 0 & 21,658780 & 11 & 10,8681017 & 10,6055 & 46,8274 & 18,457534 \\
1 & 26,550482 & 27 & 7,3709263 & 17,6082 & 51,4384 & 25,775342 \\
2 & 39,820091 & 12 & 8,3100767 & 30,6658 & 52,0658 & 38,757534 \\
3 & 55,315982 & 72 & 12,4816451 & 25,4849 & 75,4110 & 56,904110 \\
Total & 44,390995 & 122 & 17,6131134 & 10,6055 & 75,4110 & 44,052055 \\
\hline
\end{tabular}

Tabla 4. Distribución de resultados de edades en relación con el sistema de estadios de Barchilon para la primera costilla en hombres.

\begin{tabular}{|c|c|c|c|c|c|c|}
\hline $\begin{array}{c}\text { OSIFICACION } \\
\text { COSTILLA 1 }\end{array}$ & Media & N & $\begin{array}{c}\text { Desviación } \\
\text { Standard }\end{array}$ & Minimo & Máximo & Mediana \\
\hline 0 & 14,044384 & 10 & 5,4433175 & 5,3479 & 19,4274 & 15,460274 \\
1 & 29,747507 & 25 & 10,8070002 & 17,8247 & 67,7233 & 27,071233 \\
2 & 47,682636 & 37 & 11,7570961 & 24,8740 & 65,5918 & 48,345205 \\
3 & 56,080611 & 52 & 13,9889111 & 28,1205 & 75,1205 & 58,900000 \\
Total & 44,875652 & 124 & 18,0499445 & 5,3479 & 75,1205 & 45,517808 \\
\hline
\end{tabular}

Tabla 5. Distribución de resultados de edades en relación con el sistema de estadios de Barchilon para la primera costilla en mujeres.

\begin{tabular}{|c|c|c|c|c|c|c|}
\hline $\begin{array}{c}\text { OSIFICACION } \\
\text { COSTILLA 1 }\end{array}$ & Media & N & $\begin{array}{c}\text { Desviación } \\
\text { Standard }\end{array}$ & Mínimo & Máximo & Mediana \\
\hline 0 & 14,948250 & 9 & 4,7817378 & 5,3479 & 19,4274 & 17,608219 \\
1 & 29,016438 & 30 & 10,3728596 & 17,8247 & 67,7233 & 26,901370 \\
2 & 46,728767 & 23 & 11,8539409 & 24,8740 & 65,5918 & 46,827397 \\
3 & 55,346089 & 62 & 13,1161408 & 25,4849 & 75,4110 & 56,904110 \\
Total & 44,445537 & 124 & 17,8858107 & 5,3479 & 75,4110 & 44,780822 \\
\hline
\end{tabular}

Tabla 6. Distribución de resultados de edades en relación con el sistema de estadios de Barchilon para la primera costilla en lado derecho.

\begin{tabular}{|c|c|c|c|c|c|c|}
\hline $\begin{array}{c}\text { OSIFICACION } \\
\text { COSTILLA 1 }\end{array}$ & Media & N & $\begin{array}{c}\text { Desviación } \\
\text { Standard }\end{array}$ & Minimo & Máximo & Mediana \\
\hline 0 & 20,346347 & 12 & 11,3567887 & 5,3479 & 46,8274 & 18,490411 \\
1 & 26,820797 & 22 & 7,4539306 & 17,6082 & 51,4384 & 26,328767 \\
2 & 44,897576 & 26 & 11,2494872 & 24,8740 & 65,0849 & 45,939726 \\
3 & 55,927176 & 62 & 13,1538990 & 25,4849 & 75,4110 & 58,335616 \\
Total & 44,828161 & 122 & 17,7837707 & 5,3479 & 75,4110 & 45,517808 \\
\hline
\end{tabular}

Tabla 7. Distribución de resultados de edades en relación con el sistema de estadios de Barchilon para la primera costilla en lado izquierdo. 
El coeficiente de correlación de Pearson entre la edad y el sistema de estadios de Michelson y Barchilon para ambos sexos fue de 0,749, para hombres de 0,774 y para mujeres de 0,730 ( $p=0,000$ sig, dos colas).

La tabla 8 representa una tabla de contingencia de distribución de resultados de estadios en relación con las edades definidas por decenios. Los resultados de dicha tabla por decenios indican que los sujetos en estadio 0 suelen tener edades menores de 20 años de edad (80.9\%), en estadio I suelen tener menos de 30 años (94,2\%) y en estadios 2 y 3 todos los sujetos tienen más de 20 años de edad (100\%).

\begin{tabular}{|c|c|c|c|c|c|c|}
\hline & \multicolumn{4}{|c|}{ Costilla Primera } & \multirow{2}{*}{ Total } \\
\hline & & 0 & 1 & 2 & 3 & \\
\hline \multirow{7}{*}{$\begin{array}{c}\text { Grupos } \\
\text { edad }\end{array}$} & $0-20$ & 17 & 7 & 0 & 0 & 24 \\
\hline & $20-30$ & 2 & 30 & 4 & 4 & 40 \\
\hline & $30-40$ & 1 & 12 & 11 & 16 & 40 \\
\hline & $40-50$ & 1 & 0 & 15 & 24 & 40 \\
\hline & $50-60$ & 0 & 2 & 11 & 27 & 40 \\
\hline & $60-70$ & 0 & 1 & 7 & 32 & 40 \\
\hline & $>70$ & 0 & 0 & 0 & 22 & 22 \\
\hline \multicolumn{2}{|c|}{ Total } & 21 & 52 & 48 & 125 & 246 \\
\hline
\end{tabular}

Tabla 8. Tabla de contingencia entre edad estimada por grupos de edad y estadios de evolución de osificación del cartílago costal de la primera costilla según el sistema de estadios de Barchilon.

La independencia en función de la lateralidad de la costilla analizada para los estadios se comprobó mediante la prueba de Chi cuadrado de Pearson ( $\chi 2=1,827$ con 3 g.I.).

Se pudo comprobar la diferencia estadística entre sexos mediante la prueba de Chi cuadrado de Pearson ( $\chi 2=16,090$ con 3 g.l.). En las tablas de contingencia entre sexo y estadios se pudo comprobar como las mujeres tenían un predominio de estadio 2 frente a los hombres en los que predominaba el estadio 3 (tabla 9).

Tabla Contingencia Sexo - Estadio de osificación

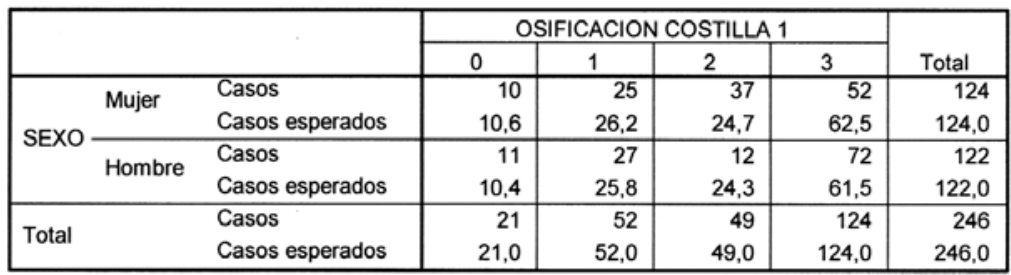

Tabla 9. Tabla de contingencia entre sexo y estadios de evolución de osificación del cartílago costal de la primera costilla según el sistema de estadios de Barchilon. Se representan los casos producidos en el análisis y los esperados en una distribución independiente.

Se realizó una prueba de retest cuatro semanas después de terminar el estudio inicial sobre I0 imágenes radiográicas de primeras costillas, siendo el coeficiente kappa estimado de I,000 para el estadiaje estimado de cada imagen. Ambas pruebas, test y retest, fueron realizadas por un único observador.

\section{DISCUSIÓN:}

Desde que en 1984 Iscan, Loth y Wright publicaron su estudio original sobre los cambios operados con la edad en la extremidad esternal de la cuarta costilla han sido múltiples los estudios 
dedicados a valorar la relación con la edad de los cambios observados osteológicamente en dicha región anatómica [7]. Varios autores han puesto a prueba la eficacia del método como predictor de la edad en distintas revistas indexadas [8,9], siendo motivo de varias tesis doctorales [ 10$]$ y estudios de validez en distintas poblaciones [ I I, I2, I3]. Se ha propuesto también la posibilidad de aplicar con cierta eficacia este método en otras costillas con la excepción de la primera [I4, I5]. No obstante, lo que ha resultado sorprendente no solo al propio Iscan sino a otros autores posteriores es el hecho de que el análisis de los cambios morfológicos con la edad de las costillas haya sido objeto de escasos estudios antes de la publicación de este método. Este fenómeno resulta aún más extraño si se considera que, con la excepción de los cartílagos laríngeos, los cartílagos costales son la única estructura anatómica que sufre un proceso de osificación progresivo que se extiende virtualmente durante toda la vida de los seres humanos.

En el caso de la primera costilla, los estudios radiológicos centrados en los cambios morfológicos con la edad han sido muy escasos y los estudios morfológicos también poco abundantes. De hecho, hasta que King en 1939 publicó su estudio sobre el proceso de osificación de los cartílagos costales persistía una discrepancia entre los autores a la hora de considerar si el proceso de osificación de este cartílago era un autentico proceso de osificación progresivo con la edad o un mero fenómeno de calcificación heterotópica relacionado con patologías respiratorias como la tuberculosis o el enfisema pulmonar [16]. El estudio de King puso de manifiesto desde una perspectiva bioquímica, histológica, anatómica y radiológica que la osificación era un fenómeno activo relacionado con la edad y con factores de stress físico determinado por los movimientos respiratorios.

En 1934, Michelson analizó los cambios morfológicos con la edad en primeras costillas observadas en radiografías de tórax postero-anteriores para lo que dispuso de un total de 5.098 radiografías de sujetos de ambos sexos y de grupos raciales caucasoide y negroide [6]. Para cuantificar los cambios morfológicos con la edad de la extremidad esternal de la primera costilla utilizó un sistema de estadios adaptado de un sistema propuesto por un autor previo para las restantes costillas [17]. Este sistema de estadios de osificación del cartílago costal definía los siguientes para la primera costilla:

- Estadio 0 ó de no calcificación ó 0\%

- Estadio I ó de calcificación mínima ó $30 \%$

- Estadio 2 ó de calcificación media ó 60 \%; en esta fase, la osificación está avanzada y casi llega a contactar con el esternón.

- Estadio 3 ó de máxima calcificación ó 90 \%; en esta fase, existe una densidad de calcificación completa que se extiende desde la extremidad costal hasta el esternón.

Los resultados de la serie de Michelson para sujetos de raza blanca se recogen en las tablas 10 y ||$[6]$

Curiosamente, en los tres decenios posteriores no se encuentran referencias en revistas indexadas sobre estudios que relacionen la osificación de la primera costilla con la edad. Los estudios centrados en el análisis antropológico de radiografías de tórax se orientaron al análisis de las diferencias de osificación intersexual de las costillas. Al parecer, fue Fischer quien por primera vez sugirió estas diferencias intersexuales en la osificación del cartílago costal [18]. Autores posteriores, como Sanders [19], McCormick, Stewart y Langford [20], Navani et al [2 I], Rao y Pai [22], mantuvieron esta línea de investigación centrada en las diferencias por sexos.

En 1965 Fully y Dehouve identificaron en estudios de radiografía de tórax PA una serie de cambios morfológicos en los cartílagos costales de todas las costillas que, de una forma rudimentaria, anteceden las características descritas por Iscan en su método osteológico ya clásico de diagnóstico de edad [23]. 


\begin{tabular}{|c|c|c|c|c|c|c|}
\hline Edad & $\begin{array}{l}\text { Estadio } \\
060 \% \\
\end{array}$ & $\begin{array}{c}\text { Estadio } \\
1630 \% \\
\end{array}$ & $\begin{array}{l}\text { Estadio } \\
2060 \% \\
\end{array}$ & $\begin{array}{c}\text { Estadio } \\
30690 \% \\
\end{array}$ & Total casos & $\begin{array}{l}\text { Promedio de } \\
\text { Calcificación }\end{array}$ \\
\hline $6-10$ & $54(100 \%)$ & 0 & $\ldots$ & $\ldots$ & 54 & 0 \\
\hline $11-15$ & $43(82,7 \%)$ & $9 \quad(17,3 \%)$ & 0 & $\ldots$ & 52 & $5,2 \%$ \\
\hline $16-20$ & $83(41,7 \%)$ & $114(57,3 \%)$ & $2(1,0 \%)$ & 0 & 199 & $17,8 \%$ \\
\hline $21-25$ & $22(8,9 \%)$ & $159(64,1 \%)$ & $66(26,6 \%)$ & $1(0,4 \%)$ & 248 & $35,6 \%$ \\
\hline $26-30$ & 0 & $112(46,5 \%)$ & $126(52,3 \%)$ & $3(1,2 \%)$ & 241 & $46,4 \%$ \\
\hline $31-35$ & 0 & $32(16,6 \%)$ & $126(65,3 \%)$ & $35(18,1 \%)$ & 193 & $60,5 \%$ \\
\hline $36-40$ & $1(0,4 \%)$ & $16(6,6 \%)$ & $143(59,3 \%)$ & $81(33,6 \%)$ & 241 & $67,8 \%$ \\
\hline $41-45$ & $\ldots$ & $7 \quad(3,4 \%)$ & $104(51,0 \%)$ & $93(45,6 \%)$ & 204 & $72,6 \%$ \\
\hline $46-50$ & $\ldots$ & $1 \quad(0,6 \%)$ & $66(42,0 \%)$ & $90(57,3 \%)$ & 157 & $77,0 \%$ \\
\hline $51-55$ & $\ldots$ & $\ldots$ & $27 \quad(30,0 \%)$ & $63(70,0 \%)$ & 90 & $81,0 \%$ \\
\hline $56-60$ & $\ldots$ & $\ldots$ & $12(25,0 \%)$ & $36(75,0 \%)$ & 48 & $82,5 \%$ \\
\hline $61-65$ & $\ldots$ & $\ldots$ & $1 \quad(7,1 \%)$ & $13(92,9 \%)$ & 14 & $87,9 \%$ \\
\hline $66-70$ & $\ldots$ & $\ldots$ & $\ldots$ & $13(100 \%)$ & 13 & $90 \%$ \\
\hline$>70$ & $\ldots$ & $\ldots$ & $\ldots$ & $4(100 \%)$ & 4 & $90 \%$ \\
\hline Total & & & & & 1.758 & \\
\hline
\end{tabular}

Tabla 10. Resultados de la serie de Michelson N para varones de raza blanca (Michelson, 1934).

\begin{tabular}{|c|c|c|c|c|c|c|}
\hline Edad & $\begin{array}{l}\text { Estadio } \\
060 \%\end{array}$ & $\begin{array}{c}\text { Estadio } \\
1030 \% \\
\end{array}$ & $\begin{array}{l}\text { Estadio } \\
2060 \% \\
\end{array}$ & $\begin{array}{c}\text { Estadio } \\
30690 \% \\
\end{array}$ & Total casos & $\begin{array}{l}\text { Promedio de } \\
\text { Calcificación }\end{array}$ \\
\hline $6-10$ & $44 \quad(100 \%)$ & 0 & $\ldots$ & $\ldots$ & 44 & 0 \\
\hline $11-15$ & $40(76,9 \%)$ & $12 \quad(23,1 \%)$ & 0 & $\ldots$ & 52 & $6,9 \%$ \\
\hline $16-20$ & $149(54,0 \%)$ & $124(44,9 \%)$ & $3 \quad(1,1 \%)$ & 0 & 276 & $41,1 \%$ \\
\hline $21-25$ & $33(16,6 \%)$ & $146(73,4 \%)$ & $20(10,0 \%)$ & 0 & 199 & $28,0 \%$ \\
\hline $26-30$ & $2 \quad(1,2 \%)$ & $113(65,3 \%)$ & $58(33,5 \%)$ & 0 & 173 & $39,7 \%$ \\
\hline $31-35$ & $1 \quad(0,6 \%)$ & $68(41,7 \%)$ & $87 \quad(53,4 \%)$ & $7(4,3 \%)$ & 163 & $48,4 \%$ \\
\hline $36-40$ & $1 \quad(0,7 \%)$ & $43 \quad(29,7 \%)$ & $96 \quad(66,2 \%)$ & $5(3,4 \%)$ & 145 & $51,7 \%$ \\
\hline $41-45$ & $\ldots$ & $21 \quad(21,4 \%)$ & $65(66,3 \%)$ & $12(12,3 \%)$ & 98 & $57,2 \%$ \\
\hline $46-50$ & $\ldots$ & $6 \quad(7,4 \%)$ & $61 \quad(75,3 \%)$ & $14(17,3 \%)$ & 81 & $63,0 \%$ \\
\hline $51-55$ & $\ldots$ & $4 \quad(11,4 \%)$ & $22(62,9 \%)$ & $9(25,7 \%)$ & 35 & $64,3 \%$ \\
\hline $56-60$ & $\ldots$ & $\ldots$ & $10(76,0 \%)$ & $3(23,1 \%)$ & 13 & $66,9 \%$ \\
\hline $61-65$ & $\ldots$ & $\ldots$ & $4 \quad(57,1 \%)$ & $3(42,9 \%)$ & 7 & $72,9 \%$ \\
\hline $66-70$ & $\ldots$ & $\ldots$ & $\ldots$ & $3(100 \%)$ & 3 & $90 \%$ \\
\hline$>70$ & $\ldots$ & $\ldots$ & $\ldots$ & $1(100 \%)$ & 1 & $90 \%$ \\
\hline Total & & & & & 1.290 & \\
\hline
\end{tabular}

Tabla 11. Resultados de la serie de Michelson N para mujeres de raza blanca (Michelson, 1934).

En 1975, Semine y Damon realizaron un estudio sobre un total de I 194 radiografías de tórax de sujetos de ambos sexos de distintos grupos geográficos (libaneses, nativos de las Islas Solomon y caucasoides norteamericanos). En estas radiografías analizaron la osificación del cartílago costal de la primera costilla mediante un sistema de estadios similar al propuesto por Michelson, aunque con cinco estadios, y la correlacionaron con la edad, el sexo y el origen geográfico de los sujetos [24]. El análisis de la osificación de la primera costilla mediante el sistema propuesto dio unos valores de coeficiente de correlación de Pearson con la edad que oscilaron entre valores de 0,42 para mujeres de la tribu Baegu y 0,77 para varones de la tribu Lau, ambos nativos de las Islas Solomon.

En 1980 Stewart y McCormick inician la publicación de una serie de trabajos relativos a la osificación de los cartílagos costales valorada radiográficamente en petos esterno-costales extraídos de cadáveres autopsiados $[25,26,27,28,29]$. Al final de esta serie de estudios, la muestra total analizada había incluido a 1989 sujetos, 1318 hombres y 67। mujeres entre 15 y 99 años de edad. Para valorar la osificación del cartílago costal de la primera costilla estos autores aplicaron un sistema de 8 estadios.

De forma análoga a los autores previos, Barrés et al en 1989 analizaron radiográficamente petos esternocostales obtenidos de cadáveres y en ellos valoraron varios parámetros, entre los cuales 
pudieron comprobar que la fusión de las esternebras, los cambios en la unión esterno-condral de las costillas y los cambios en la unión condro-costal eran los que mejor se habían correlacionado con la edad [30].

Finalmente, entre las series dedicadas al estudio radiográfico de los cambios en el cartílago costal de la primera costilla con la edad destaca el estudio publicado por Barchilon et al en 1996. Este estudio, a diferencia del precedente de Michelson de 1934, analizó una serie de sujetos mediante un diseño longitudinal, en lugar de transversal como habían hecho autores previos. Durante un periodo de 15 años los autores analizaron a 13 varones sanos a los que sometieron a radiografías seriadas con una diferencia de 2,9 años de promedio entre radiografías. La conclusión de su estudio indicaba que la progresión con la edad era evidente, aunque sometida a un ritmo de progresión individual diverso en velocidad y aspectos morfológicos en cada sujeto [5].

Recientemente, Kunos et al en 1999 han publicado un método de diagnóstico de edad basado en cambios morfológicos de la primera costilla aplicable sobre hueso. Según estos autores su método, que valora cambios estructurales en toda la anatomía de la costilla, tiene un elevado valor como método de diagnóstico de edad, con resultados comparables a los de la sínfisis pubiana, la superficie auricular del ilion o la atrición dental [3 I]. Autores posteriores, como Schmitt y Murail, sin embargo, han puesto en duda la eficacia del método propuesto por Kunos [32]. En todo caso, no deja de ser un método de interés que merecerá sin duda en un futuro nuevos estudios para valorar su eficacia como método de diagnóstico osteológico de edad.

En nuestra serie, el coeficiente de correlación de Pearson entre la edad y el estadiaje basado en el sistema de Michelson y Barchilon ha dado unos valores de correlación muy elevados, lo que indica que la asociación entre ambos factores es alta. Se ha podido observar que en ambos sexos, la existencia de una primera costilla en estadio 0 suele asociarse con edades entre 5 y 19 años, con valores extremos de hasta 46,8 años de edad. Por otra parte, los estadios de osificación 2 y 3 se han asociado con edades mínimas de 24 años de edad. De forma análoga, Michelson en 1934 ya había indicado en su serie que la edad mínima a la que los sujetos estudiados presentaban un estadio 3 de osificación era de 21 años de edad.

En consecuencia, con las salvedades inherentes a la escasez de estudios previos y la limitación de la muestra analizada para este estudio, cabe considerar que la osificación de la primera costilla puede constituir un elemento diagnóstico adicional a los ya propuestos por el AGFAD para el diagnóstico de edad en supuestos menores no identificados. La existencia de una primera costilla en estadio 3 de osificación de Michelson y Barchilon sería altamente sugestiva de una edad superior a los 21 años de edad. Por otra parte, el hecho de que la región anatómica de la unión costo-esternal de la primera costilla suela ser visible en las radiografías de tórax PA utilizadas habitualmente para el estudio de la epífisis esternal de la clavícula no exigiría la práctica de nuevos estudios radiográicos para poder valorar este parámetro de diagnóstico de edad en sujetos vivos. De este modo, no sería necesario aumentar la exposición a radiaciones ionizantes de los sujetos de estudio para poder disponer de un parámetro diagnóstico adicional [33]. Además, la posibilidad de utilizar métodos de radiografía digital para su estudio puede ser un factor añadido de limitación de la exposición radiológica a supuestos menores con fines periciales y no estrictamente terapéuticos, objetivo deseable en toda prueba aplicada a sujetos vivos con finalidad estrictamente médico legal [34].

\section{AGRADECIMIENTOS:}

A los doctores Arsenio Martínez y Elena Lángara y a los técnicos del centro de radiodiagnóstico Preteimagen de Bilbao, sin cuya desinteresada colaboración facilitándonos la muestra de radiografías utilizada en el estudio este trabajo no hubiese sido posible. 


\section{BIBLIOGRAFÍA:}

I. Schmeling A, Olze A, Reisinger W et al. Age estimation of living people undergoing criminal proceedings. The Lancet, 2001; 358(9276): 89-90.

2. Schmeling A, Geserick G, Kaatsch HJ et al. Recommendations for age determinations of living probands in criminal procedures. Anthropol Anz, 200I; 59(I): 87-9I.

3. Study Group of Forensic Age Estimation of the German Association of Forensic Medicine (200I). Guidelines for age estimation in living individuals in criminal proceedings. http://www.charite.de/rechtsmedizin/agfad/empfelung I.htm Versión en Español: http://www.justizia.net/ivml.

4. Image J versión actual 1.38 para windows. http://rsb.info.nih.gov/ij/.

5. Barchilon V, Hershkovitz I, Rotschild BM, Wish-Baratz S, Latimer B, Jellema LM, Hallel T, Arensburg B. Factors affecting the rate and pattern of the first costal cartilage. Am J Forensic Med Pathol.1996;:17(3):239-247.

6. Michelson N. The calcification of the first costal cartilage among whites and negroes. Hum Biol.1934;6:543-557.

7. Iscan MY, Loth SR, Wright RK. Metamorphosis at the esternal rib end: a new method to estimate age at death in white males. Am J Phys Anthropol.1984;65(2):147-156.

8. Russel KF, Simpson SW, Genovese J, Kinkel MD, MeindI RS, Lovejoy CO. Idependent test of the fourth rib aging technique. Am J Phys Anthropol.1993;92:53-62.

9. Dudar JC, Pfeiffer S, Saunders SR. Evaluation of morphological and histological adult skeletal age-at-death estimation techniques using ribs. J Forensic Sci.1993;38(3):677-685.

10. Reig Blanch R. Contribución a la identificación médico legal: cambios en el extremo esternal de la costilla como método complementario de determinación de la edad en el esqueleto. Tesis Doctoral. Universidad de Barcelona. 1989.

II. Yavuz MF, Iscan MY, Cologlu AS. Age assessment by rib phase analysis in Turks. Forensic Sci int. 1998;98:47-54.

12. Yoldi A. Parámetros de sexo y edad en el coxal y la articulación esterno-costal en una población mediterránea de sexo y edad conocidos. Tesis Doctoral. Universidad de Granada. 1998.

13. Oettle AC, Steyn M. Age estimation from sternal ends of ribs by phase analysis in South African blacks. J Forensic Sci.2000;45(5):107I-1079.

14. Yoder C, Ubelaker DH, Powell JF. Examination of variation in sternal rib end morphology relevant to age assessment. J Forensic Sci.2001;46(2):223-227.

I5. Loth SR, Iscan MY, Scheuerman EH. Intercostal variation at the esternal end of the rib. Forensic Sci Int.1994;65(2):135-143.

16. King JB. Calcification of the costal cartilages. Br J Radiol. 1939:12:2-12.

17. Ernts G. Fortschr. A. d. Geb. D. Röntgenstrahlen.1920;39:485-
494 (citado por Michelson N, 1934).

18. Fischer E. Verkalkungsformen de Rippenknorpel. Fortschr. a .d. Geb. D. Röntgenstrahlen u. d. Nuklearmedizin.1955;82:474-48| (citado por Navani et al. 1970).

19. Sanders CP. Sexing by costal cartilage calcification. $\mathrm{Br} J$ Radiol.1966;39:233.

20. Stewart JH, Mc Cormick WF, Langford LA. Sex determination from chest plate Roentgenograms. Am J Phys Anthropol.1985;68(2):173-195.

2I. Navani S, Shah JR, Levy PS. Determination of sex by costal cartilage calcification. Am J Roentgenol Radiother.1970;108:771-774.

22. Rao NG, Pai LM. Costal cartilage calcification pattern. A clue for estabishing sex identity. Forensic Sci Int.1988;38:193-202.

23. Fully $\mathrm{G}$, Dehouve A. Renseignements apportés dans la détermination de I'áge par I'étude anatomique et radiologique du sternum et des côtes. Annales de Médicine Légale.1965;45:469-474.

24. Semine AA, Damon A. Costochondral ossification and aging in five populations. Hum Biol.1975;47:101-II6.

25. McCormick WF. Mineralizatoon of the Costal Cartilages as an indicator of age: Preliminary observations. J Forensic Sci.1980;25(4):736-741.

26. McCormick WF, Stewart JH. Ossification of costal cartilages as an indicator of sex. Arch Pathol Lab Med.1983;107:206-210.

27. Stewart JH, Mc Cormick WF. A sex and age limited ossification pattern in human costal cartilages. Am J Clin Pathol.1984;81:765769.

28. McCormick WF, Stewart JH. Age related changes in the human plastron: a Roentgenographic and morphologic study. J Forensic Sci.1988;33(I):100-120.

29. McCormick WF, Stewart JH, Greene H. Sexing of human clavicles using length and circunference measurements. Am J Forensic Med Pathol.1991;12(2):175-181.

30. Barrés DR, Durigon M, Paraire F. Age estimation from quantitation of features of "chest plate" X-Rays. J Forensic Sci.1989;34(I):228-233.

3I. Kunos CA, Simpson SW, Russelll KF, Hershkovitz I. First rib metamorphosis: its possible utility for human age-at-death estimation. Am J Phys Anthropol. 1999;/10(3):303-323.

32. Schmitt A, Murail P. Is the first rib a reliable indicator of age at death assessment? Test of the method developed by Kunos et al (1999). Homo. 2004;54(3):207-214.

33. Schmeling A, Schultz R, Reisinger W, Mühler M, Wernecke KD, Geserick $\mathrm{G}$. Studies on the time frame for ossification of the medial clavicular epiphyseal cartilage in conventional radiography. Int J Leg Med.2004;II8(I):5-8.

34. European Commission. Radiation protection. Medico-legal exposures, exposures with ionising radiation without medical indication. Proceedings of the International Symposium. Dublin, 4-6 september 2002. European Communities. 2004. 